\title{
POR QUÉ SER JUSTOS ¿Son las normas de justicia sociales o morales?
}

\author{
WHY TO BE FAIR. \\ Are norms of justice social or moral?
}

\author{
BLANCA RodRÍGUEZ-LóPEZ bmerino@filos.ucm.es \\ Universidad Complutense de Madrid. España
}

\section{RESUMEN}

Durante los últimos años, el concepto de norma social ha sido objeto de un intenso trabajo teórico, en el que cabe destacar las aportaciones de Cristina Bicchieri y Jon Elster. Pese a las diferencias de sus respectivos planteamientos, ambos coinciden en definir el concepto por oposición a otro tipo de normas, en especial las morales. Este acuerdo no impide, sin embargo, que clasifiquen las normas de justicia de manera diferente: mientras que para Bicchieri se trata de normas sociales, Elster clasifica distintas normas de justicia en distintas categorías (sociales, cuasi morales y morales). En este trabajo, y con ayuda de algunos experimentos recientes, se analiza el caso de una norma de justicia, la norma de reparto igualitario, y se defiende que, pese a que los experimentos no son concluyentes, hay algunos factores que apuntan a la mayor validez de la teoría de Elster para el tratamiento y la conceptualización de esta norma.

\section{Palabras clave}

Bicchieri; Elster; Experimentos económicos; Justicia; Normas sociales.

\section{Abstract}

In the last few years, there has been a good amount of theoretical work around the concept of social norm. The work of Cristina Bicchieri and Jon Elster has been specially interesting and intense in this field. Although their respective approaches present substantive differences, they both agree in defining social norms in contrast with other related concepts, such as moral norms. Notwithstanding this basic agreement, they disagree when classifying norms of justice. For Bicchieri these are social norms, while Elster's view is more complex and he classifies different norms of justice in different categories: some are social while others are semi moral o moral. In this paper I focus on the treatment of one specific norm of justice, the norm of egalitarian distribution, which is a social norm for Bichieri but a moral norm from Elster's view. With the aid of some recent experiments, I conclude that Elster's view is more promising, at least when analyzing this particular norm.

\section{KEYWORDS}

Bicchieri; Elster; Economic Experiments; Justice; Social Norms. 


\section{INTRODUCCIÓN ${ }^{1}$}

Durante los últimos años, el concepto de norma social ha sido objeto de un intenso trabajo teórico, cuya finalidad es precisar un concepto que, si bien es recurrente en sociología y otras ciencias sociales afines, ha carecido de la precisión necesaria. Este trabajo ha sido acometido principalmente en tres frentes: la Teoría de la Elección Racional estándar y los últimos trabajos de C. Bicchieri y J. Elster².

Un punto en común de estos dos autores es su intento de definir la norma social señalando su diferencia específica frente a otro tipo de normas con las que, sin embargo, comparten características importantes: las normas morales. Ambos coinciden en señalar que dicha diferencia específica reside en la actitud de los agentes ante las normas y, por consiguiente, en los mecanismos mediante los cuales motivan tanto su cumplimiento como la reacción ante su violación. Se trata por tanto de una diferencia que podemos calificar de "formal" en la medida en que no es "material": no depende de su contenido. Esta visión formal puede parecer en principio extraña, puesto que por la general, y a la vista de su contenido, algunas normas, tales como las de etiqueta, son consideradas "sociales" y otras, como cumplir las promesas, "morales". Enfocar la distinción formal, con su acento en los factores motivacionales, tiene la ventaja de apuntar a un aspecto que permite identificar, y eventualmente facilitar, las circunstancias sociales bajo las cuales los agentes cumplirán o violarán las normas. Es relevante para entender el papel del contexto social en la explicación de la conducta individual y colectiva. Tal enfoque no requiere, ni mucho menos, negar que existen normas sociales y morales "prototípicas", sino únicamente el reconocimiento de que algunas normas pueden pertenecer a ambas categorías al mismo tiempo y que una misma norma puede ser social para algunos agentes y moral para otros (y quizá para el mismo agente en distintos momentos).

Pese a las diferencias notables de Bicchiery y Elster en su aproximación a las normas, ambos coinciden en un punto importante: las normas sociales (NS en adelante) son condicionales, es decir, los agentes las cumplen de una forma condicional, mientras que las normas morales (NM en adelante) son incondicionales. Podemos ver qué significa este carácter condicional de las normas sociales para cada uno de estos autores con el ejemplo de la norma relativa al cumplimiento de las promesas. Para Bicchieri, se trataría de una norma social si mi acatamiento de la misma depende de la existencia de dos tipos de expectativas (Bicchieri 2006). En primer lugar, yo debo creer que un número suficiente de individuos cumplen la norma. Se trata de expectativas empíricas. En segundo lugar, debo creer que un número suficiente de individuos esperan que yo cumpa la norma, expectativas que se califican como normativas, aunque para algunos individuos puede ser necesario que los demás no sólo esperen que yo cumpla mis trabajo.

${ }^{1}$ Agradezco a Gianluca Grimalda sus sugerencias y comentarios en el proceso de gestación de este

${ }^{2}$ Un buen análisis comparativo de las tres propuestas puede encontrarse en Tena y Güell (2011). 
promesas, sino que prefieran que lo haga y estén además dispuestos a sancionar mi conducta en caso de incumplimiento (esta condición más fuerte se recoge como Expectativas normativas con sanciones). Por su parte, Elster (2007) señala que la conformidad con las NS depende de que nuestra conducta pueda ser observada por los demás, y sea por tanto condicional a tal observación. Si yo cumplo mi promesa en la medida en que los demás, aunque solo sea el receptor de la promesa, pueden observar mi conducta de conformidad o violación de la norma, entonces para mí se trata de una NS. Si, por el contrario, cumplir las promesas es para mí una NM, ambos autores coindicen en señalar que yo cumpliré la norma de manera incondicional, es decir, con independencia de las creencias, expectativas y conducta de los demás. Bicchieri capta bien esta característica de las NM al señalar que se trata de una subclase de normas personales, como lavarme los dientes después de comer o celebrar con un benjamín el fin satisfactorio de una tarea tediosa. Cumplo o dejo de cumplir tales normas por motivos que nada tienen que ver con los demás y si me adhiero a ellas es por razones independientes de lo que mis congéneres puedan hacer, pensar, creer o esperar.

El objetivo de este trabajo es mostrar, mediante un análisis de algunos experimentos recientes, que, sin embargo, el asunto no es ni mucho menos tan sencillo. Nos centramos para ello en normas de justicia. La razón para elegir este tipo de normas, al margen de las relacionadas con el interés personal y social de las mismas (nos interesa mucho más saber por qué y bajo qué condiciones los individuos siguen o incumplen normas de justicia que porqué cumplen o dejan de cumplir normas de, por ejemplo, etiqueta), es que se trata de un caso especialmente controvertido. Por un lado, mucha gente (desde muy legos a filósofos) mantiene que las normas de justicia son NM. Más aún, para muchos la justicia es un componente esencial (a veces el más importante) de una teoría moral o política. Pero por otro lado, tanto Bicchieri como Elster (aunque este con matices, al distinguir de manera más fina entre distintas categorías de normas de justicia) las clasifican como NS. Podríamos decir que no son ni prototípicamente sociales ni morales. Si buscamos por tanto un tipo de norma que pueda ser un banco de pruebas adecuado para la capacidad explicativa de una distinción formal entre NS y NM, es muy posible que no podamos encontrar otro mejor.

\section{JUSTICIA}

Aunque la justicia ha sido desde tiempos remotos objeto de reflexión para filósofos y pensadores de diversa índole, sigue siendo objeto de controversia, a nivel normativo, qué cosas, acciones, instituciones etc. merecen ser calificadas de justas o no. A nivel empírico, aunque se ha avanzado bastante en tiempos recientes, pocas cosas están claras, salvo que no hay una única norma de justicia y que este concepto es enormemente contextual (Konow 2003). Sin embargo, a nosotros nos basta con definir el objeto de las normas de justicia (NJ en adelante) relacionadas con la adjudicación de costes y beneficios entre distintos individuos. 
Para Bicchieri, las NJ son sin lugar a dudas NS. Puesto que no hay nada intrínsecamente bueno en nuestras normas de justicia (salvo la bondad intrínseca de que haya algunas y que sean socialmente aceptadas) la razón para obedecerlas no puede residir en la norma misma. Reconoce, no obstante, que hay algo que sitúa a las NJ en un lugar especial dentro del conjunto de NS: tenernos, es cierto, una razón condicional para seguirlas pero, al menos para muchos de nosotros, esa razón condicional consiste en que consideramos que las expectativas normativas de los demás son legítimas (Bicchieri 2006: 21).

Por su parte, Elster afirma que algunas NJ son sociales mientras que otras pertenecen a una categoría especial bautizada como "normas cuasi morales", que comparten con las sociales el carácter condicional, aunque al contrario que estas no tienen efecto cuando los demás pueden observar lo que hace el agente sino cuando este puede observar lo que hacen aquellos. Los ejemplos utilizados por Elster (2009: 208 y ss.) para ilustrar la categoría de NJ que son NS están relacionados con las vigentes en el mercado laboral, relativas a la distribución de costes y beneficios entre empresarios y trabajadores.

Sin embargo, hay una NJ que Elster menciona (2006; 2007) como ejemplo de NM: la norma de reparto igualitario. Esta norma establecería que, en ausencia de consideraciones tenidas por relevantes, las cargas o beneficios han de repartirse de forma igualitaria. Por ejemplo, si los dos miembros de una pareja trabajan un número similar de horas en un trabajo similar en inversión de esfuerzo, la norma dice que deben repartir a partes iguales el trabajo doméstico. Sin embargo, si uno de los dos no trabaja (y vive del trabajo del otro), o si uno solo trabaja media jornada o si uno trabaja en una oficina y el otro en la construcción, admitimos desviaciones de la norma. Qué consideraciones se contemplan como suficientes para apartarse de la norma puede variar en diferentes contextos sociales, pero es la norma, en ausencia de aquellas, la que es calificada por Elster de moral.

La norma de reparto igualitario (NRI en adelante) es la que parece estar vigente en juegos como el Juego del dictador (JD en adelante) o el del ultimátum (JU en adelante). Ambos juegos tienen la mayor relevancia para nuestro tema, pues se han utilizado repetidamente y con distintas variantes precisamente para poner a prueba en qué medida los agentes actúan movidos por un interés egoísta puramente económico o si, por el contrario, hace falta recurrir a otras motivaciones para explicar su conducta. $Y$ entre estas otras motivaciones alternativas ocupa un lugar prominente el cumplimiento de normas. Ambos juegos son sencillos, al menos en su versión clásica, y pueden explicarse en pocas palabras.

En JU participan dos jugadores que desempeñan roles diferentes. Uno de ellos, al que se denomina "el que propone" $(P)$ recibe una determinada cantidad de dinero, digamos 10 euros, y ha de proponer al otro jugador, denominado "el que responde" (R), un determinado reparto de dicha cantidad $(x, y)$ donde $x$ es la cantidad que $P$ conservará $e$ $y$ la que recibirá $\mathrm{R}$. Si R acepta la propuesta, el reparto se efectuará del modo indicado por P. Si R la rechaza, ninguno recibirá nada. Se trata pues de una situación estratégica típica en la que el resultado depende de la acción de ambos jugadores. Tiempo atrás se 
entendió que un R motivado en exclusiva por un interés económico egoísta aceptaría cualquier oferta superior a 0 , pues de no hacerlo no obtendría nada y cualquier cosa es más que nada. Pero los experimentos realizados muestran de manera consistente un rechazo de ofertas inferiores a $(8,2)$, y estos resultados tendían a interpretarse como prueba de la falsedad de la hipótesis del individuo auto-interesado. Sin embargo, esta interpretación es como poco apresurada, tal y como hoy en día se reconoce generalmente. Lo es porque olvida que se trata precisamente de una situación estratégica y que en caso de rechazo si bien $R$ no recibe nada tampoco recibe nada $P$. $R$ tiene en sus manos un incuestionable poder de veto, que sin duda utilizará para rechazar ciertas ofertas que entiende están por debajo del límite de lo admisible, y muy probablemente $P$ anticipa este rechazo y ofrece lo que considera que está por encima de tal límite. Pero el resultado más interesante de los experimentos con JU es que la oferta modal es $(5,5)$. Es decir, típicamente los $\mathrm{P}$ ofrecen más de lo que los $\mathrm{R}$ están dispuestos a aceptar. Una de las posibles explicaciones de la conducta de $\mathrm{P}$ es que está siguiendo una $\mathrm{NJ}$, en concreto NRI.

El juego del dictador no es en realidad un juego, puesto que no plantea una situación estratégica y en él interviene un solo agente en sentido estricto (un solo individuo que toma decisiones y realiza acciones). En JD, un individuo $X$ recibe una cantidad de dinero, pongamos de nuevo 10 euros, y tiene que decidir si conserva dicha cantidad o la reparte con otro individuo $Y$, teniendo que decidir también en este último caso un determinado reparto $(x, y)$. La situación planteada hasta aquí es exactamente igual que la de JU. La diferencia es que el segundo individuo no puede sino aceptar la decisión de $X$. JD plantea una situación inmejorable para poner a prueba la conducta altruista de los individuos, al margen de cualquier consideración estratégica directa. Parece que un homo economicus tradicional conservará para sí mismo la totalidad del dinero en juego. Pero de nuevo los numerosos experimentos realizados muestran que a menudo los individuos ofrecen algo, aunque las ofertas son considerablemente menores que en JU. Aunque la conducta del dictador es tremendamente susceptible a la información que posee acerca del receptor de su posible generosidad (Aguiar, Brañas-Garza y Miller 2008), en ausencia de información muchos no ofrecen nada y la moda de ofrecimientos es del $10 \%$.

En la vida real, fuera del laboratorio, no es extraordinario encontrar individuos cuya conducta puede calificarse de altruista (aunque tampoco lo es encontrar individuos cuya conducta puede calificarse de egoísta sin ningún género de dudas). Sin embargo, tal y como ha sido señalado repetidamente (ver por ejemplo Elster 2004) una conducta aparentemente altruista puede no serlo en realidad. Puede tratarse de una conducta perfectamente egoísta (y astuta) que obedece sagazmente a consideraciones estratégicas. El agente puede, por ejemplo, estar intentando crearse una buena reputación, asegurándose la cooperación futura del receptor de su generosidad. Por ejemplo, en JD, por muy impotente que uno de los agentes sea en esa particular ocasión, pueden encontrarse en el futuro con las tornas cambiadas. Dicho de otro modo, muchas variables pueden estar actuando como causas de la conducta de manera inadvertida. Por fortuna, el laboratorio y los experimentos ofrecen precisamente un entorno en el que aislar la eficacia causal 
de distintos factores. Por eso, en los experimentos con juegos económicos en general, y con JU y JD en particular, suelen establecerse unas normas con el objeto de neutralizar las posibles consideraciones estratégicas indirectas (e.d., ajenas al planteamiento del juego). Típicamente, se utiliza el anonimato de los participantes (respecto a otros jugadores o incluso respecto al experimentador), se evitan los juegos reiterados, el emparejamiento se realiza con jugadores distintos en cada ocasión etc.

En resumen, tenemos dos juegos, JU y JD, en los cuales, y en distinta proporción, algunos jugadores se comportan de manera altruista una vez neutralizados los factores estratégicos que pueden influir en situaciones cotidianas. Una de las posibilidades es que la conducta altruista esté determinada por el cumplimiento de un NJ, y una norma plausible (aunque no la única) en las situaciones planteadas es NRI. Nuestra pregunta inicial era si podemos discriminar entre NS y NM tal y como estas han quedado definidas, y en concreto si podemos realizar esta discriminación con respecto a las NJ. Una de las respuestas más inmediatas es la que analizaremos a continuación: podemos discriminarlas mediante experimentos.

\section{EXPERIMENTOS}

Puesto que la diferencia entre NS y NM parece estar en el carácter condicional de las primeras e incondicional de las segundas, resulta útil precisar qué vamos a entender por condicionalidad/incondicionalidad:

Incondicionalidad: Si para un agente A $n$ es una NM, A actuará conforme a $n$ con independencia de la conducta y expectativas de los demás.

Condicionalidad Si para un agente A $n$ es una NS, A sólo actuará conforme a $n$ si otros cumplen $n$.

otros esperan que A cumpla $n$.

otros, con su conducta, inducen a $\mathrm{A}$ a cumplir $n$.

Estos tres modos de condicionalidad corresponden a la división de Sugden (1998), y está elegida por su claridad, pero la correspondencia con la conceptualización de Bicchieri puede hacerse fácilmente: 1 y 2 corresponden, respectivamente, a las expectativas empíricas y normativas a las que alude Bicchieri y 3 a la imposición de sanciones. Aunque no se excluyen unas a otras, al menos una debe estar presente para que pueda hablarse de condicionalidad. Para 1 y 2 , habría que precisar la cantidad y relevancia para A de los otros, pero aquí lo tomamos sin precisiones.

Antes de pasar a analizar los experimentos conviene hacer una advertencia. Puesto que se admite que una norma puede ser social para unos y moral para otros (y ambas cosas al mismo tiempo) a fortiori suponemos que una NJ está en este caso. Por tanto, suponemos que cuando se defiende que son sociales se quiere decir que lo son para la mayoría. 
Recientemente, Bicchieri ha realizado dos experimentos expresamente diseñados para este propósito. La idea que subyace a ambos es que si una norma es social, la conducta de los agentes cambiará si bajo condiciones experimentales manipulamos las expectativas. En uno de los experimentos (Bicchieri y Xiao 2009), se utiliza como herramienta el JD y en otro (Bicchieri y Chavez 2010), JU. Se trata, como hemos visto, de situaciones en la que la posible norma en juego es una NJ, normas que Bicchieri considera sociales.

Experimento 1 (E1 en adelante) El propósito de este experimento no es comprobar si las NJ son sociales o morales (cosa que Bicchieri supone: son NS) sino comprobar el peso relativo que los dos tipos de expectativas, empíricas y normativas, tienen a la hora de determinar en los agentes la conformidad con una NS, en especial cuando ambos tipos entran en conflicto. Pero para alcanzar este propósito, en E1 se manipulan, de manera directa, independiente y exógena ambos tipos de expectativas, en ocasiones en la dirección del egoísmo y en otras de la justicia.

La manipulación se efectúa del siguiente modo. Los experimentadores proporcionan a los sujetos que van a desempeñar el rol de dictador información, por otro lado verdadera y obtenida en un experimento anterior, llevado a cabo por uno de los autores (Xiao y Housser 2008), en la forma de un mensaje que resume las elecciones que los dictadores anteriores han realizado (es decir, información empírica) o/y las creencias mayoritarias acerca de lo que se debe hacer en esas circunstancias (tratándose en ese caso de información normativa). Los resultados de E1 proporcionan evidencia sustancial para mantener que las expectativas empíricas, y también, aunque con matices, las normativas, tienen un enorme potencial para predecir la conducta de los individuos. Parece mostrarse, por consiguiente, que las $\mathrm{NJ}$ son en efecto sociales, pues dependen de las expectativas y son, por ello, condicionales.

Sin embargo, si consideramos el método utilizado para la manipulación de las expectativas, los resultados no permiten tanto optimismo. El origen exógeno de la manipulación está, como hemos señalado, en los propios experimentadores. Y cualquier información que provenga de esa fuente supone un grave riesgo de que el experimento adolezca de un defecto, cuya magnitud hace que los resultados de los experimentos sean cuestionables, y que se conoce precisamente con el nombre de efecto de demanda del experimentador. Los autores son conscientes del riesgo, pero creen mitigarlo (que no eliminarlo) mediante un procedimiento de doble ciego. Me parece dudoso que ni siquiera se mitigue con esto, por la razón siguiente. El procedimiento del doble ciego, muy utilizado en experimentos económicos y que garantiza el anonimato no solo con respecto a otros sujetos que participan en el experimento sino también con respecto al experimentador, sirve para neutralizar el riesgo de que la posición de autoridad del experimentador condicione indebidamente la conducta de los sujetos. Si el problema fuera que la fuente exógena de la manipulación de las expectativas, al venir de manos del experimentador, y pese a que este asegura a los sujetos que la información proviene de otros jugadores, es vertical (puede interpretarse que procede de alguien 
que resulta ser un superior jerárquico) el doble ciego podría combatir el riesgo. Pero el problema no es este, por dos razones. La de menor importancia es que no está en absoluto claro que las expectativas tengan que ser única o prioritariamente horizontales. Más bien parece que las expectativas de distintos individuos tienen una influencia distinta en la conducta (por ejemplo, las del grupo de pares tienen más impacto en la conducta de determinados grupos de edad que la de los padres) y es perfectamente posible que en muchas ocasiones las verticales tengan mayor importancia que las horizontales. Tomando esto en cuenta, podría resultar que el doble ciego no fuera especialmente importante en este experimento en concreto.

Pero la razón más importante por la puede cuestionarse que el doble ciego siquiera mitigue el temido efecto de la demanda del experimentador se relaciona con la compresión adecuada de en qué consiste exactamente el riesgo. Es cierto que el experimentador tiene una posición de autoridad frente a los sujetos. Pero el experimentador es mucho más que eso. Es la persona que sabe, que conoce, que está como en su casa en un entorno absolutamente extraño para el sujeto, en el que este se siente perdido y, literalmente, no sabe qué hacer. Esto hace que no solo las instrucciones explícitas, sino cualquier cosa que el experimentador diga o haga pueda ser considerado por el sujeto como una "pista" sobre lo que tiene que hacer, lo que se espera de él en el contexto experimental (Zizzo 2008). Es como si mis alumnos de primero de grado, recién llegados a un entorno extraño, ante el primer trabajo que les planteo me preguntan cuántas paginas debe tener y yo les digo "no hay un número prefijado, puedes hacer lo que quieras, pero tus compañeros del curso pasado entregaron para este mismo trabajo entre 5 y 7 páginas". No hay sorpresa si me entregan ese mismo número incluso si les digo que el trabajo es anónimo y no "cuenta" para la nota. Porque no se trata solo de mi autoridad, sino de que yo soy el experto que sabe lo que es adecuado, y ellos, como recién llegados que son, no tienen ni idea. Si, tal como parece, la justicia es contextual, en un contexto que me resulta desconocido y abstracto, cualquier información que provenga del experimentador me ayudará a comprender cuál es la NJ que rige en ese contexto. Eso no significa, necesariamente, que para mí las NJ sean sociales. Puede que para mí sean morales, es decir, incondicionales, y que por tanto las siga al margen de lo que los demás hagan, crean o esperen. Pero primero tengo que saber cuál es la norma en cuestión. Y resulta sumamente cuestionable que la norma que rige la situación del experimento, en el JD, sea la norma de reparto igualitario o la de que "a quién Dios se la de, San Pedro se la bendiga". Puede que el sujeto ni siquiera esté seguro de que lo que esté en juego sea una $\mathrm{NJ}$ : bien podría ser, por ejemplo, una norma de caridad, o benevolencia o apego a los bienes materiales, que son cosas bien distintas.

Podemos por consiguiente afirmar que, en lo tocante al carácter social o moral de las $\mathrm{NJ}$, el E1 es inconcluyente.

Experimento 2 (E2 en adelante). Este experimento, que utiliza como herramienta el $\mathrm{JU}$, es notablemente más complejo que el anterior. Contrariamente a lo que sucede en 
otras ocasiones, en las que $\mathrm{P}$ puede ofrecer cualquier par $(\mathrm{x}, \mathrm{y})$, en E2 P está limitado a tres opciones: $(5,5),(8,2)$ y "moneda", opción que significa que $P$ dejará que una moneda decida la propuesta: $(5,5)$ si sale cara y $(8,2)$ si sale cruz. Las dos primeras representan opciones prototípicamente "justas" e "injustas" (las propuestas que dan al R menos de 2 son habitualmente rechazas en prácticamente todos los contextos, con algunas salvedades, de modo que podemos considerar esta opción como la que más se desvía de la NRI y responde a la vez a consideraciones estratégicas). La opción "moneda" resulta fundamental en E2, pues en este caso la manipulación de las expectativas dista de ser directa, como en $\mathrm{E} 1$, sino que depende de un diseño experimental que involucra el cruce de dos variables: información y prominencia (salience).

La primera, información, se manipula en tres condiciones:

Información plena. P elige una de las tres opciones, y si elige "moneda" el experimentador la lanza delante de R. Esto se explica por adelantado a todos los participantes, de modo que, si $P$ elige "moneda", $R$ lo sabe sin lugar a dudas.

Información privada. $\mathrm{R}$ desconoce que $\mathrm{P}$ tiene la opción de elegir "moneda", y $\mathrm{P}$ lo sabe. Se trata por tanto de un caso claro de información asimétrica.

Información limitada. Tanto $\mathrm{P}$ como $\mathrm{R}$ están al tanto de que $\mathrm{R}$ tiene las tres opciones mencionadas, pero en caso de que P elija "Moneda", esta se lanzará sin conocimiento de $R$, de modo que $R$, cuando recibe la propuesta, no sabe si es resultado de una elección directa o de "moneda".

La prominencia tiene un objetivo doble. Por un lado, se trata de subrayar la existencia de una norma y que esta se aplica a la situación planteada en el experimento. Para ello, en cualquier condición de información y antes de que tomen sus decisiones, los $\mathrm{R}$ responden un cuestionario en el que se les pide que califiquen las opciones de $\mathrm{P}$ como justas o injustas. Este objetivo no nos interesa, salvo para señalar que este procedimiento elimina en parte la indefinición de la situación que criticamos en E1. Por otro lado, en aproximadamente la mitad de las sesiones se incluye un cuestionario incentivado, tanto para los $\mathrm{R}$ como para los $\mathrm{P}$, y que también se contesta antes de tomar ninguna decisión. En el cuestionario se les pregunta a todos los participantes acerca de sus creencias respecto al porcentaje de R que señalan cada una de las tres opciones como justas. Con esto se consigue ahondar en el propósito del primer cuestionario pero, sobre todo y específicamente, se comprueba si hay un acuerdo entre las expectativas normativas de los $\mathrm{R}$ y las creencias de los $\mathrm{P}$ sobre las mismas. El objetivo de este cuestionario es fundamental para el tema que nos ocupa, pues se trata precisamente de manipular por este procedimiento las expectativas normativas. Como se trata de una manipulación que, en contraste con la de E1 podemos calificar de indirecta, es necesaria una pequeña explicación.

Una de las hipótesis del E2 es que si, en efecto, las NJ son sociales, entonces debe haber acuerdo entre las expectativas normativas de los $R$ y las creencias de los $P$ sobre ellas. Esto es sin duda cierto, pero no es suficiente. $Y$ no lo es porque tal acuerdo 
también se daría si hubiera una norma moral (compartida y establecida como tal). La existencia del acuerdo, comprobada en E2, prueba que hay una norma de justicia compartida, pero no si esta es tenida por social o moral. Además, para averiguar la existencia de una norma compartida podría haberse utilizado el segundo cuestionario en todas las sesiones. Sin embargo, es la manipulación de la prominencia y el hecho de que se utilice como variable, distinguiendo entre tratamientos con prominencia (en los que se utiliza el segundo cuestionario) y sin prominencia (en lo que no se utiliza) la que apunta directamente a nuestro objetivo. El segundo cuestionario es el único que contestan los $\mathrm{P}$ (la mitad aproximada de ellos). De este modo, se consigue que algunos $\mathrm{P}$ (los que contestan el cuestionario) enfoquen las expectativas normativas de los R. Si la NJ en juego es en efecto social, cabe esperar que en los tratamientos con prominencia haya más ofertas justas que en los tratamientos sin prominencia, algo que no sucedería si se tratara de una NM, por definición ajena a las expectativas de los demás.

Por tanto, la manipulación de la prominencia efectuada mediante el segundo cuestionario proporciona una prueba de fuego para testar el carácter social o moral de una norma, y mediante un procedimiento que escapa a los peligros de efecto de demanda del experimentador que criticamos en el caso de E1 y que parece por lo demás metodológicamente irreprochable. Sin embargo, los autores reconocen que, en este punto, sus resultados no son concluyentes. Nos olvidaremos entonces de la manipulación de la prominencia y nos fijaremos en la otra variable: la información. Pero al hacerlo nos encontramos con que esta variable no manipula las expectativas, ni directa ni indirectamente. Aunque los autores afirman que el objetivo del cambio en la información tiene el propósito de manipular las expectativas (Bicchieri y Chavez 2010: 163), en realidad ni siquiera sus predicciones están relacionadas con la manipulación de las expectativas.

Podemos recapitular diciendo que el resultado de los experimentos realizados con el propósito de probar el carácter social de las NJ (concretamente la NRI) mediante la manipulación de las expectativas en las que las NS se apoyan no es demasiado alentador: E2 solo parcialmente manipula las expectativas, cuando manipula la prominencia, con resultados inconcluyentes y E1 las manipula de un modo metodológicamente muy insatisfactorio. Naturalmente, no puede excluirse la posibilidad de que experimentos posteriores nos ofrezcan una respuesta. Sin embargo, quizá haya otro camino para decidir la cuestión.

\section{EMOCIONES}

Elster ofrece una caracterización de las normas sociales, y su contraste con las normas morales que, aunque coincide con la de Bicchieri en lo tocante al carácter condicional de las primeras e incondicional de las segundas, explica la diferencia acudiendo a las distintas emociones que la violación de unas y otras provoca tanto en el infractor como en el observador, así a las tendencias de acción correspondientes a cada una de estas emociones. Las emociones vinculadas a la violación de una NS son desprecio en el 
observador y vergüenza en el infractor, mientras que en el caso de una violación de NM encontraríamos indignación y sentimiento de culpa respectivamente. Esto parece abrir una nueva posibilidad: ¿es posible utilizar la teoría de Elster y discriminar NS y NM por las emociones asociadas? Decir que una NJ es NS equivaldría a decir que las emociones vinculadas son las propias de las NS (desprecio-vergüenza), y no las correspondientes a las NM (indignación-culpa). Pero de nuevo, al igual que en el caso de las expectativas tenemos que preguntarnos cómo podemos saberlo.

No es fácil. Elster reconoce (2009) que la diferencia entre las emociones relevantes no siempre es fácil de establecer a nivel fenomenológico. Sin embargo, hay dos procedimientos que pueden ser más fiables.

\section{Tendencias de acción}

Recurrir a las tendencias de acción correspondientes a las emociones en cuestión (evitación en el observador y ocultación en el infractor cuando se trata de NS vs. castigar-reparar daño en NM) parece una buena opción, pues estas sí son fácilmente diferenciables a nivel observacional. Desafortunadamente, existe un problema. Si bien estas acciones son fácilmente observables y difícilmente confundibles, son sin embargo difíciles de controlar experimentalmente. Esto resulta un grave inconveniente si consideramos que la observación en situaciones reales no nos permite aislar las causas de los fenómenos observados, ni neutralizar la acción de variables que suponemos irrelevantes. Por eso hacemos experimentos. Pero, dadas las condiciones de anonimato, limitación de interacciones entre los mismos sujetos y otras, que son fundamentales para controlar que la conducta altruista no tiene un componente estratégico (creación de reputación, asegurar cooperación en jugadas posteriores etc.), el observador de la violación de una NJ no puede mostrar evitación: no se puede evitar a alguien sin saber quién es. Lo que puede hacer el observador, y de hecho hace, es castigar al infractor de la norma. Pero el castigo altruista es más bien la acción correspondiente a la violación de una NM, pues es la tendencia de acción correspondiente a la indignación. Lo que sí se puede controlar (Fehr y Fischbacher 2004) es que alrededor del $60 \%$ de "observadores" en el juego del dictador castigan las distribuciones no equitativas.

Sin embargo, sería apresurado concluir que para la mayoría de los individuos (el $60 \%$ ) esta norma de justicia es una NM. Dado que el observador de la infracción no puede mostrar desprecio evitando al violador de la norma, bien pudiera suceder que decida mostrar su desprecio mediante la única opción a su alcance, el castigo, sobre todo si tenemos en cuenta que el coste del castigo para el observador no es muy grande en situaciones experimentales y que, como bien argumenta repetidamente el propio Elster, la evitación motivada por el desprecio ante la infracción de una NS tampoco le sale gratis al evitador en situaciones reales, e incluso en ocasiones le puede salir bastante cara en términos económicos. Es posible que realmente el observador sienta indignación, y sea esto lo que motive el castigo en la situación experimental, y que por 
tanto para estos observadores la NRI sea moral y no social. Pero no podemos estar seguros. Parece por tanto que tampoco por esta vía vamos a encontrar una respuesta a nuestra pregunta.

\section{El sustrato neuronal}

La segunda posibilidad consiste en recurrir al sustrato neuronal de las emociones, mediante observación con técnicas de imagen cerebral. Este ha sido el camino que ha intentado, por ejemplo, Nichols, que intenta mostrar que las NJ son morales y no sociales acudiendo a este tipo de datos (Nichols 2010). Para ello utiliza datos obtenidos en un experimento anterior (Sanfey et al. 2003), en el cual los sujetos jugaban una versión sencilla de JU, siempre en el rol de R. El objetivo de este experimento es, precisamente, analizar las reacciones cognitivas y emocionales de los individuos cuando se enfrentan a propuestas justas o injustas, escaneando su actividad cerebral durante el juego. Los sujetos del experimento no interactúan directamente con los $\mathrm{P}$ correspondientes, pero se les enseña una foto del autor de las propuestas, foto que puede corresponder a un hombre, una mujer o un ordenador.

El experimento referido por Nichols arroja dos resultados relevantes para nuestro problema. En primer lugar la imagen del escáner muestra sin lugar a dudas que las ofertas injustas generan una activación mayor de la ínsula, lo que a juicio de Nichols si bien no prueba de forma concluyente el carácter moral de la NRI sí apunta en esa dirección. Sin embargo, su optimismo parece excesivo. La ínsula es una región cerebral que se activa con cualquier emoción, de forma que lo único que parece probarse es que las emociones desempeñan un papel fundamental en el mantenimiento de las $\mathrm{NJ}$ (o al menos de la NRI), cosa que actualmente casi nadie pone en duda. Es verdad, por otro lado, que la activación de la ínsula se muestra sobre todo en la parte anterior, que está especialmente asociada a los estados y emociones negativas (típicamente, con los olores desagradables). Pero por otro lado tanto el disgusto o el desprecio como el enfado son emociones negativas. De manera que la activación de esta parte específica de la ínsula solo muestra que los sujetos experimentan una emoción negativa cuando reciben ofertas injustas, pero esto no nos ayuda a discriminar entre unas emociones negativas y otras ni, por tanto, nos ayuda a saber si para los sujetos la NRI es social o moral.

El segundo resultado relevante es que los participantes en el experimento también muestran el mismo tipo de activación de la ínsula anterior cuando la imagen que se les muestra es de un ordenador, es decir, cuando creen que la propuesta injusta procede de un maquina. De hecho, aunque los niveles de activación varían, las ofertas injustas generan actividad en la mencionada región con independencia del "género" de su contrincante: hombre, mujer u ordenador. Esto es sin duda significativo y muestra lo interiorizadas que están determinadas normas de justicia como la NRI. Pero, una vez más, no muestra si son consideradas sociales o morales.

Parece por tanto que esta segunda vía, que nos lleva a estudiar las NJ directamente a través de las emociones, aunque instructiva, tampoco nos ayuda a contestar 
nuestra pregunta. Al igual que en el caso anterior, es posible que estudios posteriores nos ayuden, pero por ahora no parecen hacerlo más que los experimentos tradicionales recogidos antes. Sin embargo, los dos experimentos analizados antes presentan algunos hallazgos que pueden ayudarnos a avanzar en nuestro problema. A ellos nos dedicaremos a continuación.

\section{QUÉ NOS DICEN EN REALIDAD LOS EXPERIMENTOS}

El experimento 2 tiene algunos resultados interesantes. En concreto, muestra la existencia de dos fenómenos.

Manipulación de la norma (MN). Cuando una norma puede interpretarse de distintas formas, los agentes la interpretarán del modo que les resulta más favorable.

El resultado más claro de los cuestionarios realizados es que en la situación planteada en E2, la opción $(5,5)$ es la considerada justa y, quizá algo más sorprendentemente, también lo es la opción "moneda" cuando esta se encuentra disponible. No importa discutir ahora las razones de los individuos para realizar este juicio normativo, sino que basta constatar el simple hecho de que es así: esta es la NJ que se considera relevante y es compartida por la mayoría de los implicados. Que ambas opciones se consideren justas lleva a los autores a predecir que habrá más elecciones de "moneda" en la condición de información plena que en las otras dos. Recordemos que en esta condición, si $\mathrm{P}$ elige "moneda" $\mathrm{R}$ lo sabe sin ningún lugar a dudas, pues la moneda se lanza en su presencia, y por tanto consideran que la elección de $\mathrm{P}$ es justa aunque al final resulte que sale cruz y por tanto el reparto es $(8,2)$ : aunque pueden considerar injusto el resultado, lo asumen como producto de un procedimiento justo (como si fuera, digamos, cosa del azar). Y, por otro lado, a los P les resulta más ventajoso en esta condición "moneda" que $(5,5)$, precisamente porque es posible que salga cruz y, si sale cara, están igual de mal en términos de interés personal que si eligen $(5,5)$ directamente.

Esta predicción se cumple y los resultados experimentales no dejan lugar a dudas: los sujetos explotan las ambigüedades de la situación en su propio interés.

Evasión de la norma $(E N)$. La predicción relevante en este caso es que habrá más elecciones de $(8,2)$ en la condición limitada que en las otras condiciones. Recordemos que en esta condición los $\mathrm{R}$ no saben si $\mathrm{P}$ ha elegido una opción directamente o si ha elegido "moneda", pero saben que esto último es posible. La predicción se cumple: en esa condición $(8,2)$ se propone más y se acepta más. La explicación salta a la vista: aunque $(8,2)$ es la opción considerada injusta en la situación, los $P$ tienen la posibilidad de comportarse injustamente sin que los $R$ lo sepan (al menos, los $R$, cuando reciben la propuesta "injusta", siempre tendrán la duda razonable acerca de si es resultado del puro azar, considerado justo). 
Ahora bien, ¿qué relación tienen estos dos fenómenos (MN y EN) con el carácter condicional de las NS? Podría parecer que MN muestra una cierta condicionalidad: puesto que cuando dos acciones distintas se consideran justas, el agente elige la que le resulta más favorable, el agente no sigue la norma de manera incondicional. Sin embargo, tal interpretación es cuestionable. Los agentes manipulan la norma precisamente para seguirla (de un modo tan beneficioso para ellos como sea posible). Y lo que hace que la manipulen no tiene relación alguna con las expectativas de los demás (expectativas que no cambian en las distintas condiciones de información) sino con la existencia de una ambigüedad en la norma, o una ambigüedad en la situación, en el sentido de que pueden aplicarse dos normas distintas.

Para mostrar una relación entre el fenómeno de MN y la condicionalidad de las NS habría que mostrar o bien que a) ambas cosas están ligadas conceptualmente 0 bien que b) de hecho se da en casos de normas sociales y no se da en casos de normas morales. Parece que esto último es lo que intentan probar Bicchieri y Chavez: que el nivel de manipulación de la norma depende de la manipulación de expectativas. Pero no lo hacen. En realidad, como vimos más arriba, no manipulan las expectativas en absoluto, sino sólo la prominencia, y esto, como ya dijimos también, o bien se daría también en el caso de una norma moral compartida o bien arroja resultados reconocidamente inconcluyentes.

Por otro lado, tenemos motivos para pensar que las normas morales no son, de hecho, ajenas a este fenómeno. Según algunos autores (Konow 2000; Nichols, 2010) tenemos razones para suponer que tales normas, pese a su carácter incondicional, pueden verse afectadas por las expectativas empíricas, en especial cuando entran en conflicto con otros deseos de los agentes (por ejemplo, con su deseo de obtener una ganancia económica). La razón es que tales expectativas pueden facilitar la operación del sesgo auto-interesado. Por ejemplo, según Nichols, podemos considerar que la conducta de los demás nos proporciona información acerca de si una determinada $\mathrm{NJ}$ se aplica o no a una situación concreta, análogamente a lo que sucede en el efecto del espectador (bystander effect). Lo que sucede con las expectativas empíricas sucederá, con mayor motivo, en el caso de las normativas: si los demás consideran que hay dos conductas igualmente justas en una situación, y una de ellas coincide con mis intereses económicos, es muy probable que yo interprete la situación de la manera que me resulte más favorable.

Estos datos prueban que hay, en efecto, una relación entre la manipulación de la norma y las expectativas, pero destruye la conexión entre ambas (el fenómeno NM y el impacto de las expectativas) y la distinción entre normas sociales y morales. La propia Bicchieri reconoce que el hecho de que una norma sea personal/moral no implica que el agente sea completamente indiferente a las expectativas, sino únicamente que el seguidor de una norma moral ofrece mayor resistencia a la influencia social en comparación con un seguidor de una norma social (Bicchieri 2010). Este reconocimiento hace que en buena medida se empiece a desdibujar la diferencia entre NS y NM establecida en términos de condicionalidad en relación a las expectativas, máxime cuando admitimos que también distintos seguidores de una NS pueden ofrecer niveles distintos de resistencia y no podemos establecer el umbral de la misma que nos hace pasar de una NS a una NM. 
Para relacionar el fenómeno de MN y la condicionalidad de las NS nos queda la alternativa de la conexión conceptual. Sin embargo, tal relación me parece extremadamente difícil de establecer. Casos como la NM (no matarás a un inocente) y la doctrina del doble efecto apuntan más bien en la dirección de la no conexión conceptual. La doctrina del doble efecto, que se remonta a Tomás de Aquino, se aplica a aquellas acciones que tienen buenos y malos efectos desde el punto de vista moral, y permite la acción (es decir, la considera moralmente admisible) siempre y cuando la intención del agente sea alcanzar el efecto positivo y el negativo (indisolublemente unido al positivo) pueda considerarse un efecto colateral no deseado. Si yo, por ejemplo, quiero calmar los dolores de un enfermo terminal o conseguir la liberación de la patria, puedo poner una inyección de morfina o una bomba en un mercado, siempre y cuando la intención directa de mi acción no sea la de matar. No es descabellado pensar que quizá, cuando además de querer el efecto positivo queremos también el negativo somos más proclives a aceptar esta doctrina y violar la norma moral en cuestión.

Mi tesis es más bien la contraria: que, en lo tocante a conexión conceptual, la manipulación de la norma está íntimamente ligada a las normas morales, y solo secundariamente a las sociales. Volveré sobre este punto más adelante.

Pasemos ahora a considerar el fenómeno de la evasión de la norma (EN). Este fenómeno tiene una indudable conexión con el carácter condicional, en la medida en que prueba que la norma evadida no se sigue sino bajo ciertas condiciones. Además, las condiciones bajo las que se cumple la norma tienen que ver directamente con los demás agentes. En concreto, con la posibilidad de que otros agentes puedan saber si la norma se viola y puedan castigar la violación. Este fenómeno conformaría lo que Bicchieri califica de "diferencia crucial" entre NS y NM: un seguidor de una NS puede ser inducido a violar la norma si sus acciones no pueden ser monitorizadas y castigadas.

Sin embargo, desde la óptica de Bicchieri, hay algo que es difícil de encajar. Desde su propia aproximación a las NS, EN solo diferenciaría a un seguidor de una NS para el que la existencia y posibilidad de aplicación de sanciones fuera determinante a la hora de seguir la norma. Por otro lado, según Bichieri, la sola existencia de expectativas empíricas y normativas sin sanciones sería suficiente en algunos casos. Entonces, habría que concluir que no podemos diferenciar en la práctica estos casos de los seguimientos de NM.

Podemos, si queremos, establecer una relación entre la condicionalidad mostrada por EN y las expectativas que entran a formar parte de la propia definición de NS, pero sólo de un modo indirecto. Parece que las expectativas, por si solas, no se relacionan con el seguimiento condicional de la norma. Deben existir, pero únicamente en la medida en que, suponiendo su ausencia, desaparecerían las sanciones: si los demás no sólo violan una norma sino que además no esperan que yo la cumpla, difícilmente castigarán mis violaciones de la misma. Parece entonces que todo se reduce a que haya o no sanciones: si un agente hace $X$ inducido por las sanciones (es decir, si deja de hacer $X$ cuando se elimina la posibilidad de ser sancionado), está siguiendo una NS. Si aún así hace $X$, está siguiendo una NM. 
Sin embargo, esto no parece muy satisfactorio, por una razón fundamental. Algunas normas que entendemos son sociales (pensemos en el ejemplo prototípico de las propinas) se cumplen en condiciones que hacen inaplicables las sanciones, como cuando acudimos solos a un local al que ni hemos ido antes ni pensamos volver. Explicamos esto en términos de sanciones "internas": la NS está tan interiorizada que el solo pensamiento de que alguien (quizá sólo el camarero) va a pensar mal de mí, me incomoda lo suficiente como para cumplir la norma. A la vista de estos casos, podemos decir que lo que el fenómeno de EN muestra es más comprehensivo. No es que la sanción no pueda aplicarse, sino que no puede aplicarse porque no puede monotorizarse. Esto es lo que sucede en su experimento: las sanciones no pueden aplicarse porque no es posible saber si la norma se ha infringido o no. Y esto es lo que no sucede en el caso de las propinas, en el que, en circunstancias normales, sí es posible saber si yo me he ajustado a la norma o la he violado. De ser así, esta sería la diferencia crucial entre NS y NM.

Podemos concluir lo analizado hasta el momento del modo siguiente. Hemos partido de una diferencia entre NS y NM en términos de las actitudes del agente: si las cumple de forma condicional o incondicional respecto a las expectativas de los demás. Pero lo que los experimentos analizados muestran (y Bicchieri reconoce) es algo mucho más débil: que la diferencia estriba en el cumplimiento condicional o incondicional respecto a la posibilidad de que se pueda monitorizar el cumplimiento o violación de la norma y, en caso de violación, se sigan sanciones. Pero esto nos aleja de la definición de Bicchieri y nos lleva cerca de Elster: las normas sociales operan cuando otras personas pueden observar lo que hace el agente.

\section{Observar y SANCIONAR}

Como vimos en un apartado anterior, para Elster lo que distingue básicamente NS y NM son las emociones asociadas a su violación. Esta diferencia en las emociones sería la diferencia primitiva, y el carácter condicional o incondicional dependería de estas. Lo que entonces no dijimos, y que a veces se olvida, es sin embargo fundamental. Lo que Elster ofrece no es una mera correspondencia entre distintos tipos de normas y las emociones correspondientes sino un esquema causal:

Violación de NS $\rightarrow$ desprecio en el observador, que $\rightarrow$ vergüenza en el actor

Violación de $\mathrm{NM} \rightarrow$ al mismo tiempo enfado o indignación en el observador y sentimiento de culpa en el actor.

Según estos esquemas, cuando un agente $\mathrm{A}$ viola una norma moral, esto provoca una determinada emoción en el observador de la violación (indignación) y otra en el violador (sentimiento de culpa), de forma simultánea. En el caso de la violación de una NS, se provoca una determinada emoción (desprecio) en el observador y solo después, y como consecuencia de la reacción del observador, aparece una respuesta emocional en el violador (vergüenza). 
Con esto a la vista, es fácil comprender el papel de los fenómenos observados por Bicchieri en su experimento así como su relación con los distintos tipos de normas. Es verdad que la aparición de EN apunta a la existencia y operación de una norma social, pues describe casos en los que el agente puede incumplir la norma sin que los demás lo sepan (o al menos sin que puedan estar seguros). Incumplir la norma en tales circunstancias evita la reacción del observador y por tanto la del agente. Es esta secuencia y esta lógica la que señala en la dirección de las NS. Si se tratara de una NM, evadir la mirada (y por tanto la reacción emocional) del observador no sirve para nada, pues la reacción emocional del agente es independiente de la del observador. $Y$ un agente no puede ocultar ante sí mismo que está violando una norma.

El seguidor de una norma moral sólo puede, en algunas circunstancias y cuando esto es posible, interpretar la norma del modo que le resulte más favorable. Esto es muy distinto de violar una norma. No puedo ocultar ante mí mismo que estoy interpretando la norma, pero en la medida en que no estoy violándola (ante mis ojos) me permito conciliar mis intereses personales y el cumplimiento de las normas morales. Tal como señalé, el fenómeno de MN, más que señalar hacia las normas sociales, señala en dirección a las morales.

La conceptualización de Elster es también extremadamente útil a la hora de explicar la relación de las sanciones con el cumplimiento de las normas. Las emociones ligadas a su violación son tales que o bien constituyen en sí mismas sanciones o bien desempeñan un papel indispensable en la aplicación de sanciones o ambas cosas.

\section{Sanciones}

Una diferencia aparente entre las teorías de Bicchieri y Elster es que Bicchieri parece sostener que sólo algunos individuos necesitan sanciones para conformarse a las NS. Sin embargo, como hemos visto, lo que sus propios experimentos prueban es que lo característico de las NS es que cuando el sujeto puede escapar a la monitorización (es decir, puede incumplir la norma sin que nadie lo sepa), lo hace.

La mencionada clasificación de Sugden clarifica esta cuestión. "Presión social" puede entenderse en términos de sanciones externas. Bicchieri tiene razón al decir que estas no son en todos los casos necesarias. Como ya indicamos antes, el propio diseño del experimento impide que el infractor de la norma esté sujeto a este tipo de sanciones.

Por su parte, Elster afirma (2009:196) que las NS también puedan caracterizarse como mandatos sustentados por sanciones. Si Elster cree que sí son en todo caso necesarias es porque incluye "sanciones internas": prototípicamente la propia vergüenza del agente. Pero conviene recordar que esta emoción, ligada a la violación de las NS, no se dispara por la propia acción transgresora, sino por el desprecio del observador. Lo que Elster presenta no es una tabla de correspondencias, sino un esquema causal. Si no hay observador, no que no lo haya de hecho, sino que no es posible que lo haya, porque es imposible que nadie sepa si yo he violado o no una norma, no hay desprecio, ni siquiera anticipado y por tanto no hay vergüenza. No hay sanción interna. La acción correspon- 
diente al desprecio (la evitación) impone una sanción externa. Pero el propio desprecio, al provocar mi vergüenza, me impone una sanción interna. Y esta puede operar ante la simple anticipación del desprecio que alguien siente hacia mí, incluso aunque yo no esté presente, porque, por ejemplo, el camarero no vea lo inadecuado de mi propina hasta que yo he salido del restaurante. Y si escapo a la monitorización, tampoco tiene lugar el sentimiento negativo (y por tanto capaz de actuar como sanción) de pensar que quizá, y contra lo previsto alguien (el camarero, otro comensal) mire el plato de la propina y tenga tiempo de expresar su desprecio antes de que me de tiempo a salir por la puerta para no volver.

Para un seguidor de una NS, estar en una situación en la que no es posible ser descubierto es incentivo suficiente para violar la norma. Esto es lo que produce el fenómeno observado de EN. La presencia de expectativas, empíricas o normativas, en condiciones normales produce como mínimo el temor ante la posibilidad de ser descubierto, y la anticipación del desprecio y la vergüenza consiguientes pueden ser suficientes para algunos sujetos. Estas son las condiciones habituales en situaciones no experimentales.

\section{Conclusiones}

Hemos partido de dos supuestos acerca de la distinción entre normas sociales y morales. En primer lugar, de acuerdo con Bicchieri y Elster, hemos establecido el contraste entre el carácter condicional de las primeras e incondicional de las segundas. A continuación, hemos supuesto la identificación de Bicchieri de la condicionalidad de las NS con su dependencia de las expectativas, tanto empíricas como normativas, para luego analizar algunos experimentos que intentan mostrar que las normas de justicia, específicamente la norma de reparto igualitario, son sociales en este sentido, es decir, dependen de las expectativas.

Sin embargo, las conclusiones sustentadas en los distintos experimentos analizados son más bien modestas. Podemos resumirlas en tres puntos:

- Las expectativas, en efecto, desempeñan un papel a la hora de discriminar NS y $\mathrm{NM}$, pero solo con muchos matices podemos decir que las NM son incondicionales. Podría decirse: si para un agente una norma es moral, no evade su cumplimiento (la cumple aunque, en caso de incumplirla, nadie se daría cuenta) pero es posible que la manipule a su favor (de modo que puede presentar el caso diciendo, quizá solo ante sí mismo, que en realidad no ha incumplido la norma porque esta no era de aplicación en el caso concreto).

- Las expectativas empíricas pueden tener un impacto tanto en NS como en NM, mediante el fenómeno de la manipulación de la norma. Por su parte, las expectativas normativas tienen impacto en las NS (y no en las NM) pero siempre a través de sanciones.

- La conceptualización de Elster parece, a la luz de los experimentos, más explicativa, 
en la medida en que el esquema causal que concreta la violación de las normas con determinadas emociones explica la conexión de los dos fenómenos de manipulación y evasión de la norma con las normas morales y sociales, respectivamente

Quisiera por último hacer un comentario sobre las normas de justicia, en concreto sobre la norma de reparto igualitario que Bicchieri considera social y Elster moral. Quizá el resultado más llamativo de los experimentos analizados es que muestran una división entre las respuestas de los sujetos. El experimento 2 muestra que cuando es posible evadir la norma, la proporción de elecciones que violan la NRI $(8,2)$ aumenta mucho (se duplica), y alcanza $58.5 \%$. Pero eso significa que $41.5 \%$ podría evadirse del cumplimiento de la norma y no lo hace. Sería no obstante apresurado concluir que para ese porcentaje la NRI es moral, pues hay otras posibles explicaciones de esta conducta (quizá, por ejemplo, creen que pese a todo el experimentador puede saber quién ha marcado $(8,2)$ directamente).

Más significativo resulta que la elección de $(5,5)$ varíe muy poco en los distintos tratamientos. Cuando es posible evadir la norma, lo elige un 35.8\%. Esto es consistente con otros experimentos que señalan que alrededor de $1 / 3$ de los sujetos hacen elecciones justas. No es muy optimista pensar que para esta proporción, la NRI es una NM. Podríamos preguntarnos si $1 / 3$ es mucho o poco o, con más precisión, si es una proporción excepcional. Pensemos en la NM por excelencia: "no matarás a un inocente" (quizá sin una justificación excelente, como salvar tu propia vida). Si fuera posible evadirse de la norma ¿cuántos lo harían? Obviamente, no hay experimentos en este sentido, pero resulta difícil creer que algo más de la mitad violaría la norma. Por otro lado, habría que comparar este porcentaje con los que violarían en condiciones similares una norma claramente social. Por ejemplo, ¿cuantos se colarían en una cola si no pudiese detectarse esa conducta? Probablemente, muchos más.

Estas comparaciones con normas sociales y morales prototípicas refuerza la idea que muchos tenemos acerca del carácter difícilmente clasificable de ciertas normas de justicia, como la NRI, y también explica por qué distintos autores tienen posiciones distintas a la hora de pronunciarse sobre su carácter. Sería interesante preguntarse la razón de este carácter, y tiendo a pensar que la respuesta no está en la "forma" de las normas de justicia, sino en su contenido y su función. Pero eso ya es otra historia.

\section{REFERENCIAS BIBLIOGRÁFICAS}

Aguiar, F., Brañas-Garza, P. y Miller, L.M. 2008. "Moral distance in dictator games." Judgment and Decision Making 3:344-354.

Bicchieri, C. 2006. The Grammar of Society, Cambridge University Press.

Bicchieri, C.y Xiao, E. 2009. "Do the right thing: but only if others do so." Journal of Behavioral Decision Making, 22:191-208. 
Bicchieri, C, y Chavez, A.K. 2010. "Behaving as expected: public information and fairness norms." Journal of Behavioral Decision Making 23:161-178.

Bicchieri , C. 2010, "Norms, preferences and conditional behavior." Politics, Philosophy and Economics, 9: 297-313.

Elster, J. 2004. "Mimicking impartiality." Pp. 112-126 en Justice and Democracy editado por K. Dowding.K,. Goodin, R. y. Pateman, C. Cambridge: University Press.

Elster, J. 2005. "Fehr on altruism, emotion, and norms." Analyse \& Kritik 27:197-211.

Elster, J. 2006. "Fairness and norms." Social Research: An International Quarterly 73: 365-376.

Elster, J. 2007. Explaining Social Behaviour. Cambridge: Cambridge University Press.

Elster, J. 2009. "Norms" Pp. 195-217 en The Oxford Handbook of Analytical Sociology, editado por Hedström y Bearman, Oxford: Oxford University Press.

Fehr, E. y Fischbacher, U. 2004. "Third-party punishment and social norms." Evolution and Human Behavior 25:63-87

Konow, J. 2000. "Fair Shares: accountability and cognitive dissonance in allocation decisions." The American Economic Review 90:1072-1091.

Konow, J. 2003. "Which is the fairest one of all? A positive analysis of justice theories." Journal of Economic Literature XLI: 1188-1239.

Nichols, S. 2010. "Emotions, norms and the genealogy of fairness." Politics, Philosophy Economics 9: 1-22.

Sanfey, A. et al. 2003. "The neural basis of economic decision-making in the ultimatum game." Science 300:1775-1758.

Sugden, R. 1998. "Normative expectations: the simultaneous evolution of institutions and norms." Pp. 73-100 en Economics, Values, and Organization, editado por Ben-Ner, A.y Putterman, L. Cambridge: Cambridge University Press.

Tena-Sánchez, J., y Güell, A. 2011. "¿Qué es una norma social? Una discusión de tres aproximaciones analíticas." Revista Internacional de Sociología 69:561-583.

Xiao, E. y Housser, D. (2008) "Emotion expression and fairness in economic exchange." Working Paper. Interdisciplinary Centre for Economic Science, Washington: George Mason University.

Zizzo, D. J. 2010 "Experimenter demand effects in economic experiments." Experimental Economics 13:75-98.

Blanca Rodríguez-López es Profesor contratado doctor en la Universidad Complutense de Madrid. Sus intereses giran en torno a cuestiones relacionadas con la epistemología de las ciencias sociales, la teoría de juegos y la filosofía política. Es autora de libros como Fuera de equilibrio. Moralidad y racionalidad indirecta (Editorial Complutense, 2008) y Locke: ensayo sobre la tolerancia y otros escritos sobre ética y obediencia civil (Biblioteca Nueva 2011)

RECIBIDO: 01/11/2011

ACEPTADO: 05/10/2012

Publicado on-line: 12/11/2012 